\title{
Anatomical landmarks for Percutaneous Transhepatic External Biliary Drainage (PTBD)
}

\author{
Udupihilla $\mathbf{J}^{1}$, Darmapala $\mathrm{A}^{1}$, Galketiya $\mathrm{KB}^{1}$ \\ ${ }^{1}$ Teaching Hospital Peradeniya/ Faculty of Medicine, University of Peradeniya
}

\begin{abstract}
PTBD is used for temporary decompression of an obstructed biliary system prior to surgical resection as it is believed to reduce major post operative complications. This is preferred over endoscopic biliary stenting(EBS)by most as EBS induces an inflammatory response making resection difficult. For palliation of malignant biliary obstruction and as treatment for common bile duct stones endoscopy is preferred. PTBD is used if endoscopy fails. In this subset internalization for malignancies and surgery for calculi are done once the patient's general condition improve.
\end{abstract}

Keywords: Percutaneous external biliary drainage, endoscopic biliary stent insertion

\section{Introduction}

PTBD is used for temporary decompression of an obstructed biliary system presenting as obstructive jaundice ${ }^{1}$.

Intrahepatic ducts coalesce to form the right and left hepatic ducts. They join to form the common hepatic duct. Once the cystic duct join it is named as the common bile duct. The obstructions requiring treatment may be at the confluence of hepatic ducts at porta hepatis or along the common bile duct up to ampulla of Vater.

PTBD may be used prior to definitive surgery ${ }^{2,3,4}$. The indication for preoperative placement is when the serum bilirubin levels are high and surgery getting delayed for more than two weeks. The need for such decompression may be urgent and life saving in the presence of cholangitis. Many surgeons prefer pre-operative decompression by external drainage as opposed to endoscopic biliary stent(EBS) placement as the latter induces inflammation making the surgery difficult ${ }^{4}$. Patients with malignancies unsuitable for curative resection need palliation. For palliation EBS is preferred1. For the patients with failed EBS, stent placement under radiological guidance is used ${ }^{1}$.

PTBD is not usually used in common bile duct stone which are best handled by endoscopy and surgery. But in the presence of severe cholangitis it may be used as a temporary measure to bring the patient out of sepsis if endoscopy fail. In bile duct/ hepatic duct benign strictures it may be used prior to surgical reconstruction. 


\section{Method}

Patients referred for PTBD were initially assessed for their fitness for the procedure. The diagnostic imaging evaluated with emphasis of the radiological anatomy of the dilated biliary system, to plan placement of drainage tube. Procedures were done at the interventional radiology suit with all precautions of sterility. Intravenous sedation and local anaesthesia were used. Patients were monitored continuously. In all patients external drains were placed via the left lobe of liver. Ultrasound and fluoroscopy were used to identify ductal anatomy while placing. With ultrasound guidance, appropriate segmental duct is punctured. Site of puncture is kept peripheral as possible as more central puncture has higher risk of major vascular injury.

After successful puncture, a guide wire is passed through the puncture needle. During the procedure, check cholangiograms are done periodically to follow the biliary anatomy. Then the external drainage catheter is left in the biliary system for its decompression.

\section{Results}

A total number of 38 patients

underwent the procedure. 22 males and 16 females. The age distribution was 32 to 84 years.

The procedure was performed for following indications (Table 1).

All patients with malignancies following PTBD were treated by definitive surgery, two to three weeks later. The patient with failed PTBD had a peri-ampullary carcinoma and peripheral duct dilatation was inadequate for successful puncture. He underwent pancreatico-duodenectomy early.

Two with common bile duct stones presented with acute cholangitis and required PTBD as EBS failed. The patient with a choledochal cyst was a thirty two year old lady in the first trimester of pregnancy. To bridge until delivery decompression EBS failed and required PTBD. Pregnancy continued until 34 weeks when she had a pre term delivery and was subjected to resection of choledochal cyst and Roux-N-Y hepatico-jejunostomy. Two patients

Table 1: number of patients undergoing PTBD for different indications

\begin{tabular}{|l|l|l|l|}
\hline Indication & Pre-operative & Palliative & Number of patients \\
\hline Hilar cholangiocarcinoma & 5 & 3 & 8 \\
\hline Head of pancreas carcinoma & 8 & 1 & 9 \\
\hline Peri-ampullary carcinoma & 15 (with 1 failure) & 1 & 16 \\
\hline Common bile duct stone & 2 & & 2 \\
\hline Common bile duct stricture & 2 & & 2 \\
\hline Choledochal cyst & 1 & & 1 \\
\hline Total & 33 & 5 & 38 \\
\hline
\end{tabular}


with bile duct strictures underwent reconstruction after two weeks from the placement of PTBD. The five patients needing palliative PTBD were after failed EBS. One of them with a pancreatic head neoplasm developed a bile leak in to the peritoneal cavity and was managed with laparotomy and cholecysto-jejunostomy.

\section{Discussion}

PTBD is used as pre-operative decompression or for palliation in malignancies as discussed in the introduction $^{1,2,3,4}$. The use in benign pathologies are limited. In our series it has been in used two patients with acute cholangitis with stone disease and another two with a benign stricture and a choledocahal cyst respectively. Two with stones and one with choledochal cyst underwent PTBD as endoscopic stent insertion failed. The two with bile duct strictures had elective placement of EBS for pre-operative decompression.

The need for pre-operative biliary decompression is a matter of debate ${ }^{2,3}$. In a recent systematic review, it has been shown to reduce complications following resections ${ }^{3}$. The length of hospital stay and mortality rates have not shown a statistically significant difference ${ }^{3}$. EBS is associated with an inflammatory reaction more than $\mathrm{PTBD}^{4}$. In our unit all patients planned for curative resections are referred for PTBD except in few patients showing minimal duct dilatation.
For palliation in malignancy EBS is preferred to $\mathrm{PTBD}^{1}$. In case of EBS failure PTBD and later internalization is performed. This happens more often in hilar occlusions than in distal obstructions ${ }^{1}$. In our series five patients required PTBD after failed EBS and three of them were hilar cholangiocarcinomas.

In PTBD access in to ducts is possible from left or right side and often happens on personal preference ${ }^{(1)}$. But when advantages and disadvantages are compared left approach is more beneficial $^{(1)}$.

\section{Conclusion}

PTBD is useful for pre-operative decompression and palliation whenEBS fails. The anatomy of the obstructed duct system as well as related surface and internal anatomy are important for successful placement.

\section{Conflict of interest}

None declared.

\section{Correspondence}

Kuda B Galketiya, Consultant Surgeon and Professor in Surgery,

Teaching Hospital Peradeniya/ Faculty of Medicine, University of Peradeniya, Peradeniya.

E-mail: kbgalketiya@yahoo.com 


\section{References}

1. SH Chandrashekhara, $\mathrm{S}$

Gamanagatti, Anuradha Singh, and Sushma Bhatnagar Current Status of Percutaneous Transhepatic Biliary Drainage in Palliation of Malignant Obstructive Jaundice: A Review Indian J Palliat Care. 2016 Oct-Dec; 22(4): 378-387 doi: 10.4103/0973-1075.191746

2. Takada T Is preoperative biliary drainage necessary according to evidence-based medicine $\mathrm{J}$ Hepatobiliary Pancreat Surg. 2001;8(1):58-64.
3. Harsha Moole, Matthew Bechtold, and Srinivas R. Puli Efficacy of preoperative biliary drainage in malignant obstructive jaundice: a meta-analysis and systematic review World J Surg Oncol. 2016; 14: 182.

4. Kwang Min Kim, ${ }^{*}$ Ji Won Park, $\dagger$ Jong Kyun Lee, $\uparrow$ Kwang Hyuck Lee, $\dagger$ Kyu Taek Lee, $\dagger$ and Sang Goon Shim A Comparison of Preoperative Biliary Drainage Methods for Perihilar Cholangiocarcinoma: Endoscopic versus Percutaneous Transhepatic Biliary Drainage Gut Liver. 2015 Nov; 9(6): 791-799. 